\title{
Área superficial de carbones activados y modificados obtenidos del recurso agrícola Saccharum officinarum
}

\author{
Surface area of activated and modified charcoals obtained from \\ agricultural resources Saccharum officinarum
}

\author{
Fredy Colpas C. ; Arnulfo Tarón D. ${ }^{2}$; Rafael González C. ${ }^{3}$
}

1 Docente, Ph.D. Universidad de Cartagena, Cartagena, Colombia, fcolpasc1@unicartagena.edu.co.

2 Docente, Ph.D. Universidad de Cartagena, Cartagena, Colombia, atarond@unicartagena.edu.co.

3 Docente, Ph.D. Universidad de Cartagena, Cartagena, Colombia, rgonzalezc1@unicartagena.edu.co.

Citar: Colpas, F.; Tarón, A.; González, R. 2017. Área superficial de carbones activados y modificados obtenidos del recurso agrícola Saccharum officinarum. Rev. Cienc. Agr. 34(2): 62 - 72. doi: http://dx.doi. org/10.22267/rcia.173402.72.

Recibido: Julio 19 de $2016 . \quad$ Aceptado: Marzo 19 de 2017.

\section{RESUMEN}

El carbón activado es un material utilizado industrialmente en adsorción de gases, filtrados, limpieza de líquidos y como soporte para catalizadores en medios no oxidantes. Además tiene varias aplicaciones ambientales como en la adsorción de iones metálicos de plomo. El objetivo de este trabajo fue la obtención de carbones activados carbonizando biomasa de caña de azúcar a $400^{\circ} \mathrm{C}$ en atmósfera de nitrógeno, activando con ácido fosfórico y oxidando con ácido nítrico o peróxido de hidrógeno seguido de tratamiento térmico. La preparación de carbones activados a partir de residuos agrícolas está desarrollándose con excelentes resultados potencialmente aplicables. Bajo este contexto, se muestra un método de preparación mediante activación química con ácido fosfórico, la cual aumentó el contenido de carbono y disminuyó el de oxígeno, seguidamente con el objeto de estudiar el desarrollo del área superficial se somete a proceso de oxidación con calentamiento térmico. Los carbones obtenidos fueron caracterizados mediante análisis proximal y para la determinación de los grupos funcionales oxigenados se utilizó la espectroscopia infrarroja de reflectancia difusa-transformada de Fourier. La determinación de grupos oxigenados básicos y ácidos se realizó por el método Boehm. Para la medición delárea superficial se utilizó el método BET. El área de microporos se incrementó por el tratamiento con $\mathrm{HNO}_{3} \mathrm{y} \mathrm{H}_{2} \mathrm{O}_{2}$ desde $278 \mathrm{~m}^{2} / \mathrm{g}$ hasta 402 
y $446 \mathrm{~m}^{2} / \mathrm{g}$, respectivamente. La mayor parte del área en los carbones se debió a los microporos. El $\mathrm{pH}$ de los carbones fue aproximadamente de tres y los grupos $\mathrm{COOH}$ alcanzaron valores hasta $2,12 \mathrm{meq} / \mathrm{g}$.

Palabras clave: superficie específica, subproducto agrícola, carbonización, método de Boehm.

\begin{abstract}
Activated charcoal is a material used in industry for absorbing gases and filtrates, cleansing liquids as well as for supporting catalysts in non-oxidative media. In addition, it is utilized in several environmental applications, such as in the absorption of metal lead ions. The objective of this work was the obtainment of activated charcoals by carbonizing sugarcane biomass at $400^{\circ} \mathrm{C}$ in a nitrogen atmosphere through activation with phosphoric acid and by oxidization with nitric acid or hydrogen peroxide followed by heat treatment. The preparation of activated charcoals from agricultural waste is being developed with excellent and potentially applicable results. In this context, a preparation method is shown using a chemical activation with phosphoric acid, which increased the content of carbon and decreased that of oxygen, then in order to study the development of surface area, it was put through an oxidation process with thermal heating. The charcoals obtained were characterized by proximal analysis, and diffuse reflectance infrared spectroscopy with Fourier transform was used for the determination of oxygenated functional groups. Basic oxygenated groups and acids were determined by means of Boehm's method, while the BET method was used for the measurement of the surface area. The area of micropores was increased by treatment with $\mathrm{HNO}_{3}$ and $\mathrm{H}_{2} \mathrm{O}_{2}$ from $278 \mathrm{~m}^{2} / \mathrm{g}$ up to 402 and $446 \mathrm{~m}^{2} / \mathrm{g}$, respectively. Most of the area on the charcoals was due to micropores. Charcoal pH was approximately 3 and $\mathrm{COOH}$ groups reached values of up to $2.12 \mathrm{meq} / \mathrm{g}$.
\end{abstract}

Key words: specific surface, agricultural byproduct, carbonization, Boehm's method.

\section{INTRODUCCIÓN}

El carbón es una mezcla heterogénea de plantas remanentes y minerales asociados que sufrieron cambios físicos y químicos originados por procesos biológicos y geológicos. Se compone químicamente por oxígeno, hidrógeno, carbono, nitrógeno, azufre y materia mineral (Piñerez et al., 2009). El carbón activado es un material muy utilizado en la industria, en laboratorios químicos y en medicina, además de varias aplicaciones ambientales; en la industria se usa para la adsorción de contaminantes (Primera et al., 2011; Costa et al., 2017) filtrado y limpieza de fluidos, purificación de aguas (Colpas et al 2015; Cao et al., 2017) y retención de metales preciosos en los laboratorios químicos se usa como fase estacionaria para separación cromatografía y soporte para catalizadores en medios no oxidantes (Fraga et al., 2009) en medicina se usa para el tratamiento de envenenamientos y sobredosis $\mathrm{y}$ tiene aplicaciones ambientales tales como remediación en casos de vertimientos tóxicos accidentales. Los carbones activados se obtienen de materiales con alto contenido de carbón como carbones, petróleo y desechos vegetales o animales mediante tratamientos químicos o físicos que incrementan su porosidad de manera que puedan adsorber una 
gran cantidad de sustancias (Yahya et al., 2015; Primera et al., 2011).

Los carbones industriales son producidos en su mayoría a partir de materiales carbonosos naturales como carbón y material de desecho vegetal como madera, semillas y podas. Los materiales de partida para obtener carbón activado también pueden ser: algas, cáscara de arroz, lignina, aserrín, cáscara de semilla de algodón, lignito, lodos ácidos de petróleo, madera, carbón mineral, huesos de frutas, melaza, carbón vegetal, desperdicios de pasta de papel, negro de humo, residuos de carne y sangre, olotes, huesos, desechos de destilería, pescado, cereales, cuesco de palma africana, turba, carbohidratos, coque de petróleo, granos de café, hollín, cáscara de nueces, grafito (Caglayan y Aksoylu, 2013), cáscara de coco (Song et al., 2010), cáscara de frutas (Tongpoothorn et al., 2011) y residuo de tabaco (Kilic et al., 2011). Estas materias primas deben tener alto contenido de carbono y bajo contenido de material mineral, y ser de fácil activación, alto rendimiento, baja degradación durante su almacenamiento y bajo costo.

El tratamiento usado para preparar el carbón activado y el material precursor afectan las características del producto final tales como porosidad, área superficial y tipo de grupos funcionales superficiales (Primera et al., 2011). Por su bajo costo, las sustancias más usadas para la activación química de carbones son el hidróxido de sodio y el ácido fosfórico y además, porque imparte adecuadas propiedades texturales y estructurales a los productos. Usualmente, la activación química de carbones se lleva acabo a menos de $900^{\circ} \mathrm{C}$ en atmósfera de nitrógeno (Tzvetkov et al., 2016).
El estudio del desarrollo del área superficial durante la preparación de carbones activado es de vital importancia para determinar la importancia del material de partida, fijar los parámetros a utilizar para obtener las características del área deseada y decidir la aplicación del producto obtenido.

\section{Superficie específica y porosidad de las} partículas de carbón activado. La fisisorción de nitrógeno es la forma más universalmente conocida, validada y aceptada para la determinación de la superficie específica de las partículas de carbón mineral. Además, en función de la naturaleza físico-química del material y del rango de tamaños de la porosidad presente, esta técnica permite detectar los poros, evaluar su morfología, conectividad y distribución de tamaños, dentro del rango de validez del método, determinado por la existencia o no de los fenómenos físico-químicos en los que se basa el cálculo asociado a ella (García y Moreno, 2007; Palmira y Cauca, 2007).

\section{Superficie específica, superficie externa} e interna. Se denomina superficie específica al área de la superficie por unidad de masa $\left(\mathrm{m}^{2} / \mathrm{g}\right)$. Constituye la suma del área de la superficie de todas las partículas que forman un gramo de material (Amaringo y Hormaza, 2013). En el caso de los carbones activados, la superficie de las partículas presenta una alta reactividad físico-química con el medio, lo que implica la existencia de fenómenos como la adsorción de cationes y moléculas de agua o variación de la carga eléctrica y de las fuerzas de atracción-repulsión entre partículas en función del $\mathrm{pH}$, salinidad y temperatura del medio. Cuando se realiza un análisis de adsorción de nitrógeno, se pone en contacto las partículas de carbón activado con el gas nitrógeno. 
La presión se va incrementando poco a poco mediante dosis programadas y las moléculas de nitrógeno responden al incremento de presión fijándose a la superficie de las partículas y de las paredes de los poros accesibles. Una vez se ha cubierto la totalidad de la superficie con moléculas de nitrógeno, comienzan a llenarse los poros de menor tamaño y finalmente puede tener lugar (o no) el fenómeno de la condensación capilar, transformando el gas en líquido en el interior de los poros en condiciones de presión cercanas a la presión de saturación (Castrillón et al., 2012). Acorde con lo anterior, el objetivo de este trabajo, se centró en la obtención de carbones activados a partir de bagazo de caña y la evaluación de la activación para la adsorción de materiales contaminantes.

\section{MATERIALES Y MÉTODOS}

Carbonización del material de partida. La biomasa de $S$. officinarum recolectó en la central de abastos del mercado de Bazurto (Cartagena-Colombia). La biomasa se secó a $105^{\circ} \mathrm{C}$ durante 24 horas y posteriormente se trituró. Para la carbonización se parte de $500 \mathrm{~g}$ de material seco, en fracciones de $100 \mathrm{~g}$, luego fueron calentadas en atmosfera de nitrógeno hasta $400^{\circ} \mathrm{C}$, con una rampa de $10^{\circ} \mathrm{C} / \mathrm{min}$ y se mantuvo en esta condición por $1 \mathrm{~h}$. El material fue codificado como BC carbonizado. Los carbonizados obtenidos a partir de la biomasa de $S$. officinarum se molieron y se tamizaron a un tamaño de partícula de 125 a $300 \mu \mathrm{m}$ utilizando tamices con números de mallas 50 y 120 .

Obtención de carbones activados. El carbón obtenido se impregnó con ácido ortofosfórico al 21\% p/v con agitación constan- te por 4,5h; el carbón resultante se secó a $105^{\circ} \mathrm{C}$ por $24 \mathrm{~h}$, se calentó con una rampa de $10^{\circ} \mathrm{C} / \mathrm{min}$ en atmósfera de nitrógeno empelando un flujo de $110 \mathrm{~mL} / \mathrm{min}$ hasta $450^{\circ} \mathrm{C}$ y manteniendo ésta condición por $2 \mathrm{~h}$. Finalmente, el material activado fue lavado con agua destilada caliente y fría hasta que la conductividad de las aguas de lavado fuera muy similar a la conductividad del agua destilada, y luego se procedió a secarlas a una temperatura de $105^{\circ} \mathrm{C}$ por un lapso de tiempo de 12 horas (Rodríguez-Reinoso y Molina-Sabio, 1998; Huidobro et al., 2001). El material de partida se codificó como BC y el carbón activado como BC-A.

Tratamiento térmico y oxidación. A partir de BC-A se sintetizaron dos nuevos materiales. BC-A se calentó a $600^{\circ} \mathrm{C}$ en atmósfera de nitrógeno $(110 \mathrm{~mL} / \mathrm{min})$ por $1 \mathrm{~h}$ y seguidamente se oxidó con ácido nítrico $\left(\mathrm{BC}-\mathrm{HNO}_{3}\right)$ o peróxido de hidrógeno $\left(\mathrm{BC}-\mathrm{H}_{2} \mathrm{O}_{2}\right)$. Para el tratamiento con ácido nítrico, $20 \mathrm{~g}$ de BC-A, se mezclaron con $250 \mathrm{ml}$ de $\mathrm{HNO}_{3}$ al $15 \%$ por $0,5 \mathrm{~h}$; la mezcla se filtró, se lavó con agua destilada y se secó a $105^{\circ} \mathrm{C}$ por $24 \mathrm{~h}$; posteriormente, el carbón activado seco se calentó en atmósfera de nitrógeno (flujo de $110 \mathrm{~mL} / \mathrm{min})$ hasta $600^{\circ} \mathrm{C}\left(10^{\circ} \mathrm{C} / \mathrm{min}\right)$ y se mantuvo a estas condiciones por $1 \mathrm{~h}$. Para el tratamiento con peróxido de hidrogeno, $20 \mathrm{~g}$ de BC-A se mezclaron con $250 \mathrm{ml}$ de $\mathrm{H}_{2} \mathrm{O}_{2}$ al $10 \%$ por $0,5 \mathrm{~h}$; la mezcla se filtró, se lavó con agua destilada y se secó a $105^{\circ} \mathrm{C}$ por $24 \mathrm{~h}$; posteriormente, el sólido seco se calentó en atmósfera de nitrógeno (flujo de $110 \mathrm{~mL} /$ min) desde $30^{\circ} \mathrm{C}$ hasta $600^{\circ} \mathrm{C}\left(10^{\circ} \mathrm{C} / \mathrm{min}\right)$ y se mantuvo a estas condiciones por $1 \mathrm{~h}$ (Shamsijazeyi y Kaghazelin, 2010). 
Caracterización de los materiales. La caracterización de los carbonizados, carbones activados y carbones activados modificados se basó en los siguientes análisis: Análisis próximo, análisis último, análisis por espectroscopia infrarroja con transformada de Fourier con reflectancia difusa (DRIFTS), $\mathrm{pH}$ de las suspensiones acuosas, análisis textural realizando isotermas de adsorción de nitrógeno y determinación de grupos oxigenados ácidos y básicos utilizando el método Boehm (Boehm, 1984; Primera et al., 2011).

Análisis próximo. En este método se determinó el porcentaje de humedad, materia volátil, cenizas y carbono fijo por diferencia de todos los materiales obtenidos. Para este análisis se utilizaron las normas ASTM 3302, ASTM 3175, ASTM 3174 y ASTM 3172 (ASTM, 2004).

Análisis último. $\mathrm{BC}$ y $\mathrm{BC}-\mathrm{A}$ fueron caracterizados a través de análisis último usando la norma ASTM-D3178-89 (ASTM, 2002). En esta caracterización, la determinación de carbono e hidrógeno se realizó por pirolisis de los carbones en un sistema cerrado, determinando los productos de la combustión, dióxido de carbono y agua, después de la oxidación completa. El carbono y el hidrógeno se determinaron por el incremento de peso de trampas para $\mathrm{CO}_{2} \mathrm{y} \mathrm{H}_{2} \mathrm{O}$. En este proceso, el nitrógeno presente en los sólidos se convirtió en sales de amonio por digestión con ácido sulfúrico concentrado en una mezcla catalizada con sulfato de potasio a alta temperatura. Estas sales se disolvieron en una solución alcalina caliente de la cual el amonio se evaporó y se determinó por titulación ácido-base colectándolo en un recipiente con una cantidad medida de ácido clorhídrico. Para la determinación de azufre total de los carbones, se utilizó el método de la bomba lavadora SCFI. En este método, los carbones se calcinaron a $800^{\circ} \mathrm{C}$ por $1,5 \mathrm{~h}$ con una mezcla de óxido de magnesio y carbonato de bario que precipita el azufre como $\mathrm{BaSO}_{4}$ a partir del cual se calcula el contenido de este elemento (SCFI, 1982).

El porcentaje de oxígeno se calculó por diferencia, utilizando la siguiente expresión:

$$
100-\sum(\% \text { de } \mathrm{C}+\% \text { de } \mathrm{H}+\% \text { de } \mathrm{N}+\% \text { de } \mathrm{S})
$$

Análisis textural de los materiales obtenidos. Las medidas de área superficial específica y de microporosidad se obtuvieron a través de la adsorción de nitrógeno a $77 \mathrm{~K}$ por el método BET y por el t-plot, usando un equipo Micromeritics Gemini 2375. Previo a la adsorción del nitrógeno, las muestras fueron desgasificadas a $300^{\circ} \mathrm{C}$ hasta una presión de $2 \mathrm{~mm}$ de Hg. La adsorción se efectuó en el intervalo de valores de $\mathrm{p} / \mathrm{p}^{\circ}$ de 0,01 a 1 .

Determinación de grupos oxigenados ácidos. Para la determinación de los grupos carboxílicos se usó el método de Boehm (1994). En estos análisis se pesaron $\sim 0,5 \mathrm{~g}$ de cada carbón y se agitaron a temperatura ambiente por 24 horas con $50 \mathrm{~mL}$ de una solución de $\mathrm{NaOH}$ 0,1 N. Luego se filtró el carbón y la solución se tituló potenciométricamente con

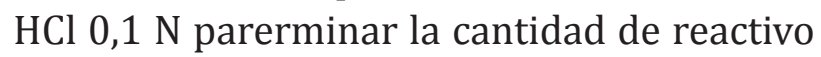
consumido.

Determinación de grupos oxigenados básicos. Se pesaron aproximadamente 0,5g de cada material y se colocaron en agitación constante a temperatura ambiente durante 24 horas con $50 \mathrm{~mL}$ de ácido clorhídrico 0,1N. Luego se filtró y la solución se tituló con $\mathrm{NaOH} 0,1 \mathrm{~N}$ usando titulación potenciométrica (Boehm, 1994; Primera et al., 2011). 


\section{Determinación del pH de los materiales} obtenidos. Para la determinación del pH generado por los carbones en solución acuosa, se mezcló 1,0g de cada carbón con 20,0mL de agua destilada libre de $\mathrm{CO}_{2}$ en recipientes de vidrio y se agitaron a temperatura ambiente por 24 horas; luego, se midió el pH de las suspensiones acuosas con un electrodo de vidrio (Orion 940) (Castilla et al., 2000).

\section{RESULTADOS Y DISCUSIÓN}

Durante la carbonización de la biomasa de caña de azúcar, las unidades de celulosa, principal componente de los materiales vegetales de partida, sufren depolimerización transformándose en unidades más pequeñas, se desprende materia volátil y se produce un sólido más rico en carbono (Rodríguez-Reinoso y Molina-Sabio, 1998). La Tabla 1 muestra resultados del análisis último del material vegetal de partida y del carbón activado con ácido ortofosfórico.

El proceso de carbonización aumentó significativamente el contenido de carbono de BC del 47 al 74\% (BC-A) al tiempo que se disminuyó la cantidad de oxígeno e hidrógeno. Este aumento del contenido de carbono se debe al carácter deshidratante del ácido fosfórico, que facilita la pérdida de hidrógeno y oxígeno en forma de agua, proceso que ocurre simultáneamente con la compactación de unidades carbonadas grandes a unidades más pequeñas y enriquecimiento en carbono.

La biomasa de caña original está compuesto básicamente por lignina, celulosa y hemicelulosa. Con la carbonización a $400^{\circ} \mathrm{C}$, solo la celulosa $\mathrm{y}$ hemicelulosa se depolimerizan en unidades más pequeñas para dar origen a una gran masa rica en carbono, ya que la lignina se descompone a temperaturas mayores; sin embargo, la impregnación con el ácido fosfórico facilita la hidrólisis de la lignina que queda presente después del proceso de carbonizado, lo que conduce al aumento de propiedades texturales como el área superficial específica y la porosidad (Gao et al., 2016). Todos estos fenómenos de rompimiento de estructuras conducen de igual forma al aumento de la aromaticidad del carbón, la pérdida del carácter alifático y la formación de una estructura rígida entrecruzada (Gao et al., 2016).

La Tabla 2 muestra los valores del área superficial específica de los materiales activados. Se observa que en el carbón activado únicamente con ácido ortofosfórico, la mayor parte del área total es atribuida a la presencia de microporos, lo cual se debe al carácter deshidratante del mismo, el cual promueve la descomposición de grupos oxigenados principalmente.

Tabla 1. Resultados del Análisis Elemental de la biomasa de caña original y activado con ácido ortofosfórico.

\begin{tabular}{cccccc}
\hline \multicolumn{5}{c}{ Análisis último (en base seca) } \\
\hline Material & C (\%) & H (\%) & N (\%) & S (\%) & O (\%) \\
\hline BC & $47,1 \pm 0,1$ & $6,9 \pm 0,1$ & 0,770 & $0,09 \pm 0$ & $45,1 \pm 0,1$ \\
\hline BC-A & $74,0 \pm 0,2$ & $3,6 \pm 0,1$ & $0,57 \pm 0,08$ & $0 \pm 0$ & $21,8 \pm 0,2$ \\
\hline
\end{tabular}

BC= carbón no activo, BC-A= carbón activado. 
Tabla 2. Resultados obtenidos para la caracterización textural y acidez de los carbones activados.

\begin{tabular}{lccccc}
\hline Carbón & $\begin{array}{c}\text { Área de } \\
\text { microporos } \\
\left(\mathbf{m}^{2} / \mathbf{g}\right)\end{array}$ & $\begin{array}{c}\text { Área } \\
\text { externa } \\
\left(\mathbf{m}^{2} / \mathbf{g}\right)\end{array}$ & $\begin{array}{c}\text { Área total } \\
\left(\mathbf{m}^{2} / \mathbf{g}\right)\end{array}$ & $\begin{array}{c}\text { Grupos } \\
\mathbf{C O O H} \\
(\mathbf{m e q} / \mathbf{g})\end{array}$ & $\begin{array}{c}\mathbf{p H ~ d e} \\
\text { Carbones }\end{array}$ \\
\hline BC-A & $278,3 \pm 0,1$ & $90,5 \pm 0,3$ & $368,7 \pm 0,1$ & $1,68 \pm 0,05$ & $2,9 \pm 0,1$ \\
${\text { BC- } \mathbf{H}_{2} \mathbf{O}_{2}}$ & $446,4 \pm 0,1$ & $92,8 \pm 0,5$ & $539,2 \pm 0,2$ & $2,12 \pm 0,02$ & $2,9 \pm 0,2$ \\
BC-HNO $_{3}$ & $401,7 \pm 0,3$ & $57,6 \pm 0,2$ & $459,3 \pm 0,3$ & $1,68 \pm 0,05$ & $3,2 \pm 0,1$ \\
\hline
\end{tabular}

Esta descomposición estimula la formación de canales a través de los cuales, el agua formada por la acción del ácido emerge del carbón, originando así la formación de los microporos. En el caso de los carbones activados oxidados con ácido nítrico y peróxido de hidrogeno, la mayor parte del área superficial especifica total también se atribuye, en gran proporción, a los microporos presentes; sin embargo, estos carbones presentaron mayores áreas, lo cual se debe, a que éstos agentes químicos son oxidantes fuertes que conducen a la descomposición de parte de la estructura carbonácea a óxidos de carbono $\left(\mathrm{CO}_{2}\right.$ principalmente), originando nuevos poros que aumentan la microporosidad y el área total.

El estudio de los grupos funcionales oxigenados presentes en las muestras activadas reveló que no existen grupos con carácter básico. La Tabla 2 también muestra que el contenido de grupos $\mathrm{COOH}$ aumenta con la oxidación con peróxido de hidrogeno, mientras que el ácido nítrico no contribuye con este parámetro. El pH de las suspensiones acuosas de los carbones fue ácido $(2,9$ a 3,2), lo cual significa que estos carbones poseen una predominancia de grupos funcionales ácidos en su superficie, importantes para el proceso de adsorción de metales (Colpas et al., 2015). Según la Figura 1, las isotermas que resultaron fueron tipo I, los cuales son características de carbones microporosos, típico de carbones activados.

El rango de presión relativa durante el cual se adsorbe el nitrógeno esta entre 0,02 y $0,1 \mathrm{P} / \mathrm{P}^{\circ}$. Según la Tabla 3, los valores de área de Lagmiur son mayores debido esencialmente al fenómeno de quimisorción que se tiene en cuenta en estos procesos, los cuales conllevan a la adhesión de las moléculas en la monocapa aumentando de esta manera el número de partículas cubiertas sobre la superficie del adsórbato.

Para la biomasa de $S$. officinarum activado con peróxido de hidrogeno, el rango de presión relativa esta entre 0,02 y $0,1 \mathrm{P} / \mathrm{P}^{\circ}$ para la adsorción de nitrógeno, igualmente para el análisis de Lagmuir la presión absoluta esta entre 150 y 300 unidades. Con respecto a los cálculos de área de microporos, para Harkin-Jura, Halsey, Broekoff-de Boer, son relativamente iguales. El área de microporos para el bagazo de caña activado y bagazo de caña modificado con tratamiento térmico según Halsey y Broekoff de Boer presentan resultados similares (Tabla 3). 


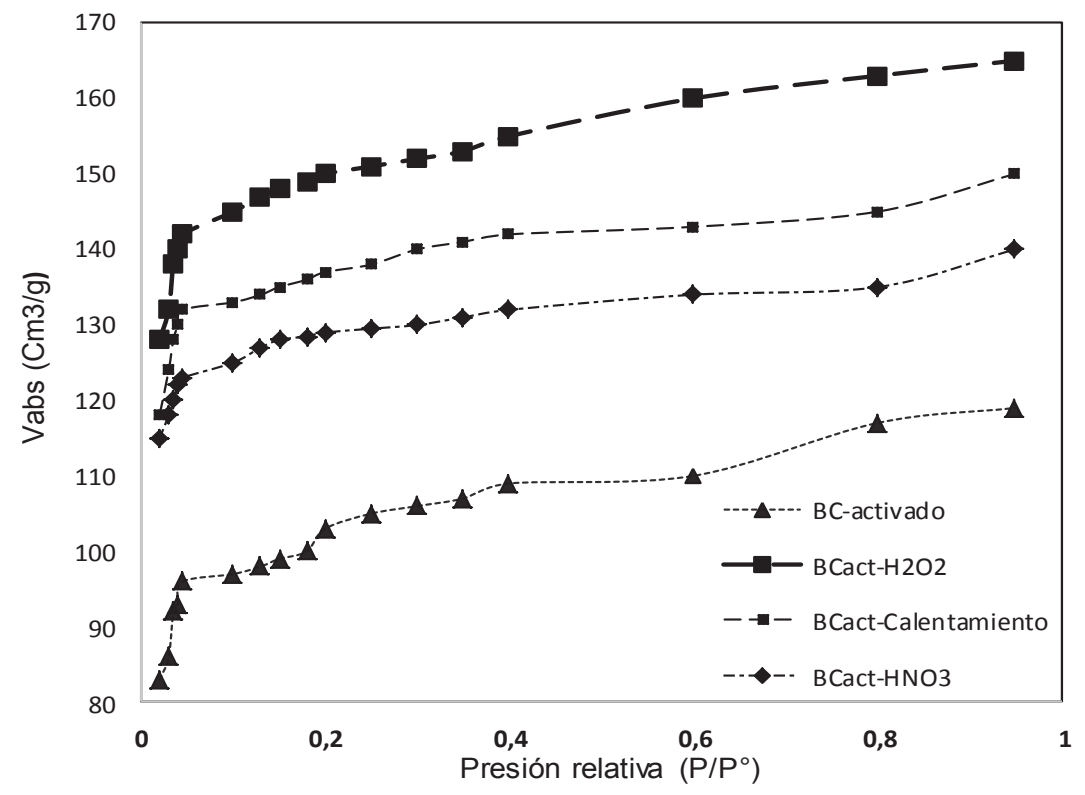

Figura 1. Isotermas de Adsorción de los diferentes materiales.

Tabla 3. Medida de área superficial de los materiales por los métodos BET, Langmuir, Halsey, Broekoff de Boer y Harkin-Jura*

\begin{tabular}{|c|c|c|c|c|c|}
\hline Muestra & $\begin{array}{c}\text { Área } \\
\text { BET } \\
\left(\mathrm{m}^{2} / \mathrm{g}\right)\end{array}$ & $\begin{array}{c}\text { Área } \\
\text { Langmuir } \\
\left(\mathrm{m}^{2} / \mathrm{g}\right)\end{array}$ & $\begin{array}{c}\text { Área } \\
\text { Microporos } \\
\text { Harkin-Jura } \\
\left(\mathrm{m}^{2} / \mathrm{g}\right)\end{array}$ & $\begin{array}{c}\text { Area } \\
\text { Microporos } \\
\text { Halsey }\left(\mathrm{m}^{2} / \mathrm{g}\right)\end{array}$ & $\begin{array}{l}\text { Área Microporos } \\
\text { Broekoff-de } \\
\text { Boer }\left(\mathrm{m}^{2} / \mathrm{g}\right)\end{array}$ \\
\hline Bagazo de caña activado & 378,9 & 490,9 & 301,4 & 284,2 & 298,9 \\
\hline $\begin{array}{l}\text { Bagazo de caña modificado con } \\
\text { calentamiento }\end{array}$ & 519,7 & 635,5 & 456,5 & 442,4 & 454,5 \\
\hline $\begin{array}{l}\text { Bagazo de caña modificado con } \\
\mathrm{HNO}_{3}\end{array}$ & 485,1 & 585,5 & 434,6 & 485,1 & 433,0 \\
\hline $\begin{array}{l}\text { Bagazo de caña modificado com } \\
\mathrm{H}_{2} \mathrm{O}_{2}\end{array}$ & 569,3 & $700, .0$ & 488,2 & 470,2 & 485,6 \\
\hline
\end{tabular}

*(Carvalho y Zapata, 2015; Jusoh et al., 2011; Dada et al., 2012; Bordoloi et al., 2017). 


\section{CONCLUSIÓN}

Se obtuvieron carbones activados a partir de biomasa de $S$. officinarum. El proceso de activación aumentó significativamente el contenido de carbono de la biomasa de caña. El aumento del área ocasionado por el tratamiento con ácido nítrico y peróxido de hidrogeno se debe al carácter oxidante de estas sustancias, las cuales descomponen partedelaestructura carbonosa, estimulando la formación de nuevos poros. Los carbones activados poseen una predominancia de grupos funcionales ácidos en su superficie, importantes para el proceso de adsorción de metales, especialmente aquellos que tengan un radio iónico proporcional al tamaño de los poros. Así mismo, las isotermas de adsorción de todos los materiales fueron tipo I (BET).

\section{REFERENCIAS BIBLIOGRÁFICAS}

1. Amaringo, A.; Hormaza, A. 2013. Determinación del punto de carga cero y punto isoeléctrico de dos residuos agrícolas y su aplicación en la remoción de colorantes. Revista de Investigación Agraria y Ambiental. 4(2):27 - 36 .

2. Arenas, E.; Chejne, F. 2004. The effect of the activating agent and temperature on the porosity development of physically activated coal chars. Carbon. 42(1213):2451 - 2455. doi: doi.org/10.1016/j. carbon.2004.04.041.

3. ASTM D3178-89 - American Society for Testing and Materials. 2002. Standard Test Methods for Carbon and Hydrogen in the Analysis Sample of Coal and Coke (Withdrawn 2007). En: https://www.astm.org/ Standards/D3178.htm; consulta: mayo, 2016.
4. ASTM - American Society for Testing and Materials. 2004. Anual Book of ASTM. Standards, Volume 01.08. American Society for Testing and Materials, West Constohocken. 713p.

5. Boehm, H.P. 1994. Some aspects of the surface chemistry of carbon blacks and other carbons. Carbon. 32 (5):759 - 769. doi: doi. org/10.1016/0008-6223(94)90031-0.

6. Bordoloi, N.; Goswami, R.; Kumar, M.; Kataki, R. 2017. Biosorption of Co (II) from aqueous solution using algal biochar: Kinetics and isotherm studies. Bioresource Technology 244:1465 - 1469. doi: doi. org/10.1016/j.biortech.2017.05.139.

7. Caglayan, B.; Aksoylu, B. 2013. $\mathrm{CO}_{2}$ adsorption on chemically modified activated carbón. J. Hazard. Mater. 252:19 - 28. doi: doi. org/10.1016/j.jhazmat.2013.02.028.

8. Carvalho, C.; Zapata, C. 2015. Thermodynamic analysis of sorption isotherms of dehydrated yacon (Smallanthus sonchifolius) bagasse. Food Bioscience 12:26 - 33. doi: doi.org/10.1016/j.fbio.2015.07.001.

9. Castilla, M.; López-Ramón, M.V.; CarrascoMarín, F. 2000. Changes in surface chemistry of activated carbons by wet oxidation. Carbon. 38(14):1999 - 2001. doi: doi. org/10.1016/S0008-6223(00)00048-8.

10. Cao, Y.; Wang, K.; Wang, X.; Gu, Z.; Ambrico, T.; Gibbons, W.; Fand, Q.; Talukder, T. 2017. Preparation of active carbons from corn stalk for butanol vapor adsorption. Journal of Energy Chemistry. 26:35 - 41. doi: http://dx.doi.org/10.1016/j. jechem.2016.08.009.

11. Castrillón, M.; Giraldo, L.; Moreno, J. 2012. Carbones activados obtenidos a partir de residuos de llantas con diferente tamaño de partícula. Afinidad LXIX. (45):266 - 271.

12. Colpas, F.; Tarón, A.; Fong, W. 2015. Analisis del desarrollo textural de carbones activados preparados a partir de zuro de maíz. Rev. Temas Agrarios. 20(1):105 - 114. 
13. Costa, P.; Alves, J.; Azevedo, D.; Bastos, M. 2017. Preparation of biomass-based activated carbons and their evaluation for biogas upgrading purposes. Industrial Crops \& Products. 109:134 - 140. doi: doi. org/10.1016/j.indcrop.2017.08.017.

14. Dada, A.O.; Olalekan, A.P.; Olatunya, A.M.; Dada, 0. 2012. Langmuir, Freundlich, Temkin and Dubinin-Radushkevichn isotherms studies of equilibrium sorption of $\mathrm{Zn} 2^{+}$unto phosphoric acid modified rice Husk. IOSR J. Appl. Chem. 3(1):38 - 45.

15. Fraga, M.A.; Jordao, E.; Mendes, M.J.; Freitas, M.M.A.; Faria, J.L.; Figueiredo, J.L. 2009. Properties of carbon-supported platinum catalysts: role of carbon surface sites. J. Catal. 209:355 - 364. doi: doi. org/10.1006/jcat.2002.3637.

16. Gao, Y.; Xu, S.; Ortaboy, S.; Kan, Y.; Yue, Q.; Gao, B. 2016. Preparation of well-developed mesoporous activated carbon with high yield by ammonium polyphosphate activation. Journal of the Taiwan Institute of Chemical Engineers. 66:394 - 399. doi: doi.org/10.1016/j.jtice.2016.06.037.

17. García, V.; Moreno, J. C. 2007. Caracterización superficial en fase gas y líquida de carbones activados. Revista de Ingeniería. $27: 1-16$

18. Huidobro, A.; Pastor, A.C.; Rodríguez-Reinoso, F. 2001. Preparation of Activated Carbon Cloth from Viscous Rayon. Part IV. Chemical Activation. Carbon. 39(3):389 - 398. doi: doi.org/10.1016/S00086223(00)00131-7.

19. Jusoh, A.; Hartini, W.J.H.; Ali, N., Endut, A., 2011. Study on the removal of pesticide in agricultural runoff by granular activated carbon. Bioresour. Technol. 102:5312 5318. doi: doi.org/10.1080/19443994.20 13.826329.
20. Kilic, M.; Apaydin-Varol, A.; Pütün. 2011. Adsorptive removal of phenol from aqueous solutions on activated carbon prepared from tobacco residues: Equilibrium, kinetics and thermodynamics. J Hazard Mat. 189 (1-2):397 - 403. doi:doi. org/10.1016/j.jhazmat.2011.02.051.

21. Piñerez, J.; Barraza, J.; Blandon, M. 2009. Constantes cinéticas de flotación del grupo maceral vitrinita de dos carbones colombianos. Rev ing e inv. 29(3):29 - 35.

22. Palmira, V.; Cauca, V. 2007. Caracterización morfológica del carbonizado de carbones pulverizados : estado del arte. Revista Facultad de Ingeniería. (41):84 - 97.

23. Primera, O.; Colpas, F.; Meza, E.; Fernández, R. 2011. Carbones activados a partir de bagazo de caña de azúcar y zuro de maíz para la adsorción de cadmio y plomo. Rev Acad Colomb Cienc. 25(136):387 - 396.

24. Rodríguez-Reinoso, F y Molina-Sabio, M. (1998). Carbones activados a partir de materiales lignocelulósicos. Química e Industria.45(9):563-571

25. SCFI. Secretaría de Comercio y Fomento Industrial. 1982. Norma mexicana NMX-B-158-1982, métodos para determinar el azufre total en la muestra de carbón y coque.En: http://200.77.231.100/ work/normas/nmx/1982/nmx-b-1581982.pdf. ; consulta: mayo, 2016.

26. Shamsijazeyi, H.; Kaghazchi T. 2010. Investigation of nitricacid treatment of activated carbon for enhanced aqueous mercury removal. J Ind Eng Chem. 16(5):852 - 858. doi: doi.org/10.1016/j.jiec.2010.03.012.

27. Song, X.; Liu, H.; Chenga, L.; Qu. Y.2010. Surface modification of coconut-based activated carbon by liquid-phase oxidation and its effects on lead ion adsorption. Desalination. 255(1-3):78 - 83. doi: doi. org/10.1016/j.desal.2010.01.011. 
28. Tongpoothorn, W.; Sriuttha, M.; Homchan, P.; Chanthai, S.; Ruangviriyacha. C. 2011. Preparation of activated carbon derived from Jatropha curcas fruit shell by simple thermochemical activation and characterization of their physicochemical properties. Chem Eng Res Des. 89(3):335 - 340. doi: doi.org/10.1016/j.cherd.2010.06.012.

29. Tzvetkov, G.; Mihaylova, S.; Stoitchkova, k.; Tzvetkov, P.; Spassov, T. 2016. Mechanochemical and chemical activation of lignocellulosic material to prepare powdered activated carbons for adsorption applications. Powder Technology. 299:41 - 50. doi: doi.org/10.1016/j.powtec.2016.05.033.

30. Yahya, M.; Al-Qodah, Z.; Ngah, C. 2015. Agricultural bio-waste materials as potential sustainable precursors used for activated carbon production: A review. Renewable Sustainable Energy Review. 46: 218 - 235. doi: doi.org/10.1016/j.rser.2015.02.051 